\title{
Big Data in the Aerospace Industry
}

\author{
Victor Emmanuell BADEA ${ }^{1}$, Alin ZAMFIROIU ${ }^{1,2}$, Radu BONCEA ${ }^{1,3}$ \\ ${ }^{1}$ National Institute for Research and Development in Informatics - ICI \\ ${ }^{2}$ The Bucharest University of Economic Studies, Bucharest, Romania \\ ${ }^{3}$ University Politehnica of Bucharest, Faculty of Electronics, Telecommunications and \\ Information Technology \\ emmanuell.badea@ici.ro,zamfiroiu@ici.ro,radu@rotld.ro
}

This paper presents the approaches related to the need for large volume data analysis, Big Data, and also the information that the beneficiaries of this analysis can interpret. Aerospace companies understand better the challenges of Big Data than the rest of the industries. Also, in this paper we describe a novel analytical system that enables query processing and predictive analytics over streams of large aviation data.

Keywords: Big Data, SAP - Predictive Maintenance, Predictive Maintenance of Propulsion Systems for Aircraft, Big Data in aerospace.

1 Introduction

The diffusion of digital technologies in people's everyday life has stimulated humanto-human interactions between man and machine-machine. These interactions generate massive volumes of data, commonly referred to as "Big Data" (BD), and characterized by rapid growth of large volumes of complex datasets that exceed the abilities of commonly known data management systems.

Due to the need to give more value to businesses, companies have begun to adopt Big Data as a solution, migrating from classical databases and data stores that lack flexibility and are not optimized enough. Large data is a collection of such large and complex datasets that it becomes difficult to process using database management tools or traditional data processing applications.

The concept addresses large volumes of data automatically generated from different autonomous data sources. Given that traditional databases have reached limits on analyzing these data, dedicated solutions should be considered. In recent approaches, important data is characterized by principles known as volume, variety, speed and $4 \mathrm{~V}$ veracity. Challenges include capturing, storing, searching, sharing, analyzing, and viewing. Such data, high volume, high-speed information and / or wide-ranging information requires new forms of processing to enable improved decision-making, knowledge discovery and process optimization. 


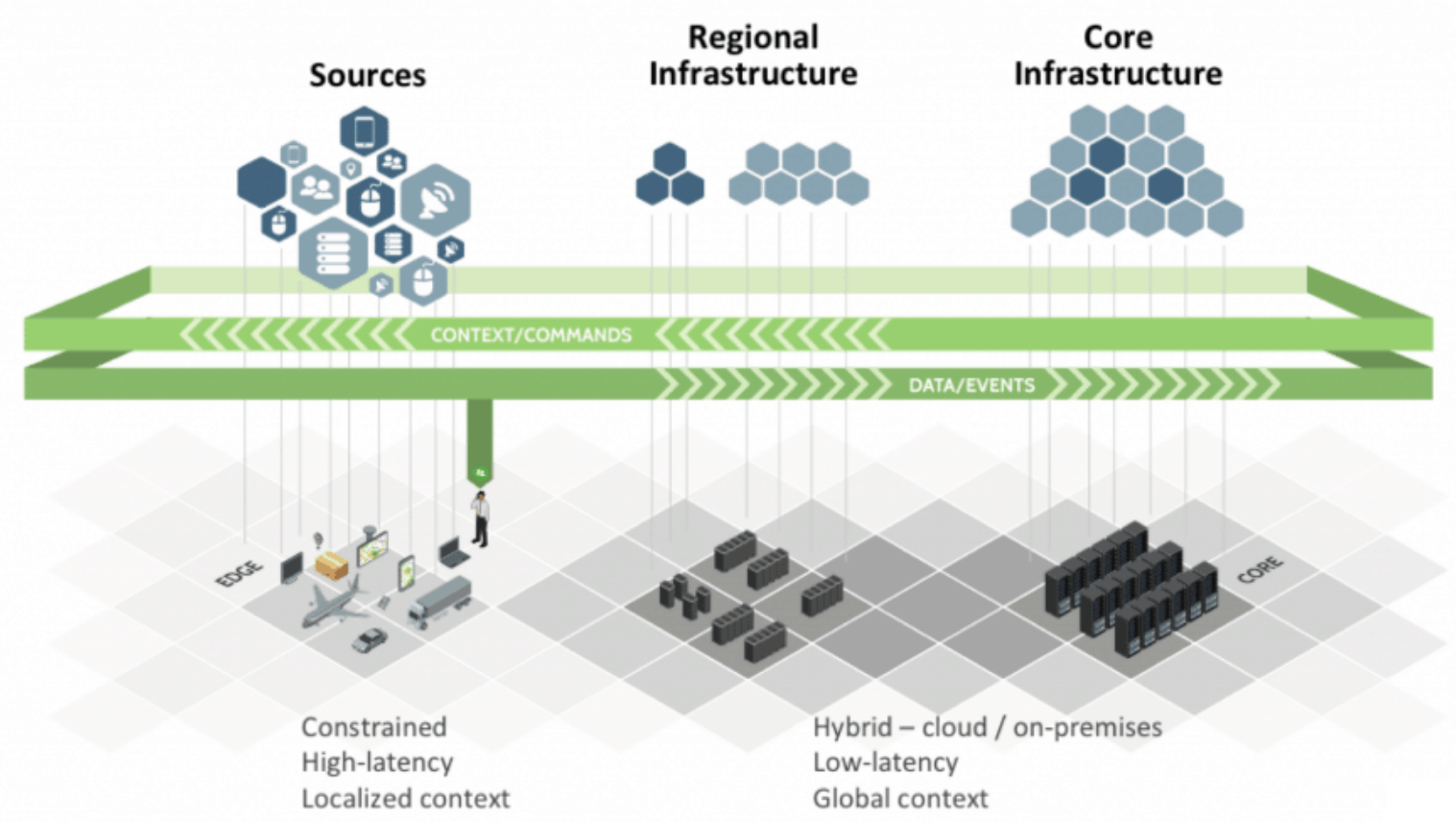

Fig. 1. Big Data int IoT

The IoT implies that data is collected from several types of sources in constrained environments. Large-scale data generated in the IoT offers as an immediate promise realtime responses and predictions and personalized options.

\section{Aerospace Industry}

Big Data represents a new generation of technologies and architectures designed to extract value from the huge data volumes with a large variety, allowing processing and analysis in real time. [1]

Aerospace companies understand better the challenges of Big Data than the rest of the industries.

A clear example of the impact of Big Data in the aerospace industry is the "digital aircraft" from our days, which can collect up to 300,000 parameters, depending on the duration of the flight and the type of aircraft. A major aircraft manufacturer estimates that data transmission will increase by $14 \%$ over the next few years.

A simple and practical example could be the following: An average Boeing 737, twoengine commercial aircraft, takes a six-hour flight between New York and Los Angeles. Each engine generates 20 terabytes of information per hour. By multiplying 20 terabytes of information from a single engine with a 6 hour flight, we will get 240 terabytes of data from the engines. According to the National Association of Air Traffic Controllers in the United States, a total of 87,000 are crossing the sky of the United States of America, of which are 29,000 commercial flights. By multiplying this by 365 days, we will get a real challenge, but also a simplification in terms of Big Data service. [2] 


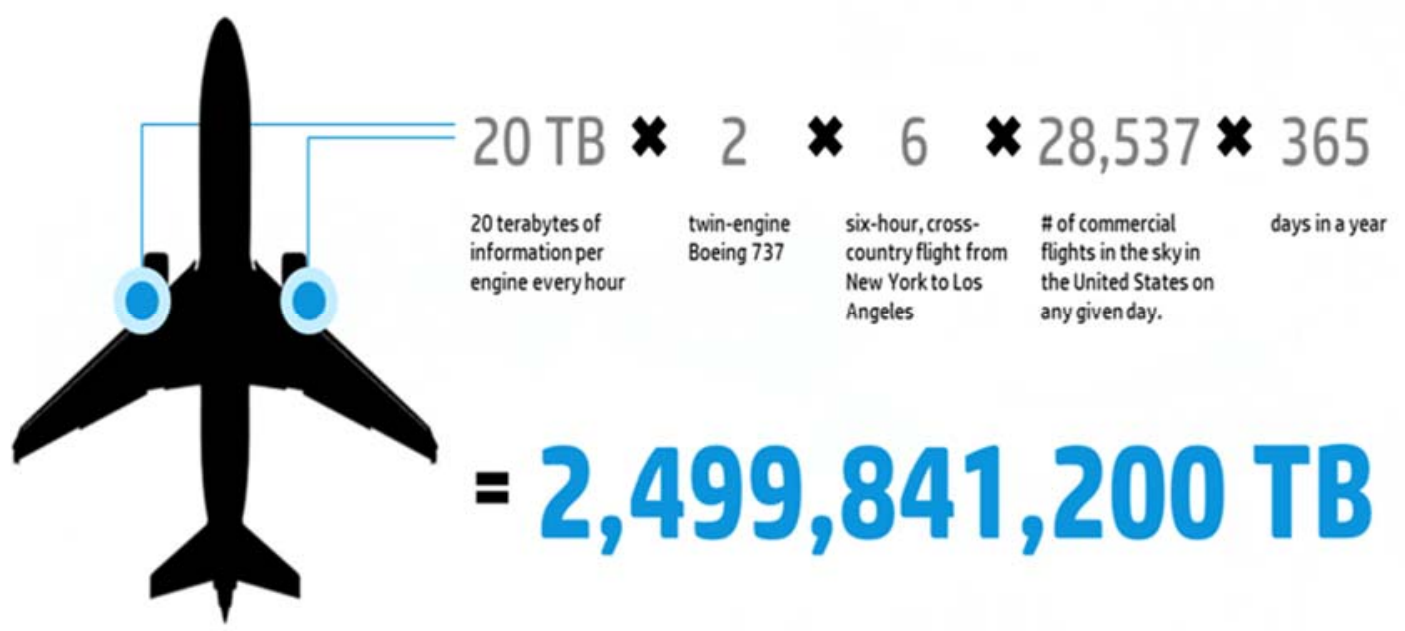

Fig. 2. Sensors data from a cross-country flight [2]

To better understand the magnitude of this amount of data, we will make the following comparison: the number of ten digits represented in the Figure 2 in $T B$, mentioned above, is converted to $2.7 \mathrm{ZB}$. This $2.7 \mathrm{ZB}$ is almost equal to the total global traffic data estimated in 2015. [2]

The analysis of this information results in the following questions:

- Why an aircraft manufacturer/ commercial airline operator would like to receive all the data collected and stored?

- What would be the impact if this information were not used?

The answer would be very simple: an aircraft manufacturer/ airline operator would normally use this data to maximize operational efficiency, and the impact of not using this information would particularly affect the company's operations, aircraft production, maintenance and management company for the level of the air fleet.

When an airline buys a 100-390 million dollar aircraft for its fleet, the goal is to keep the airplane in service for at least 18 hours a day for the next 15 to 20 years. At the heart of this effort is an airline maintenance organization that aims to manage routine and non-routine issues to keep the fleet up and running.

Based on an analysis of the International Air Transport Association (IATA), the main cause of $42 \%$ flight delays is based on airline processes such as maintenance. For every hour when the aircraft is out of service, the commercial air operator loses an average of $\$ 10,000$. [2]
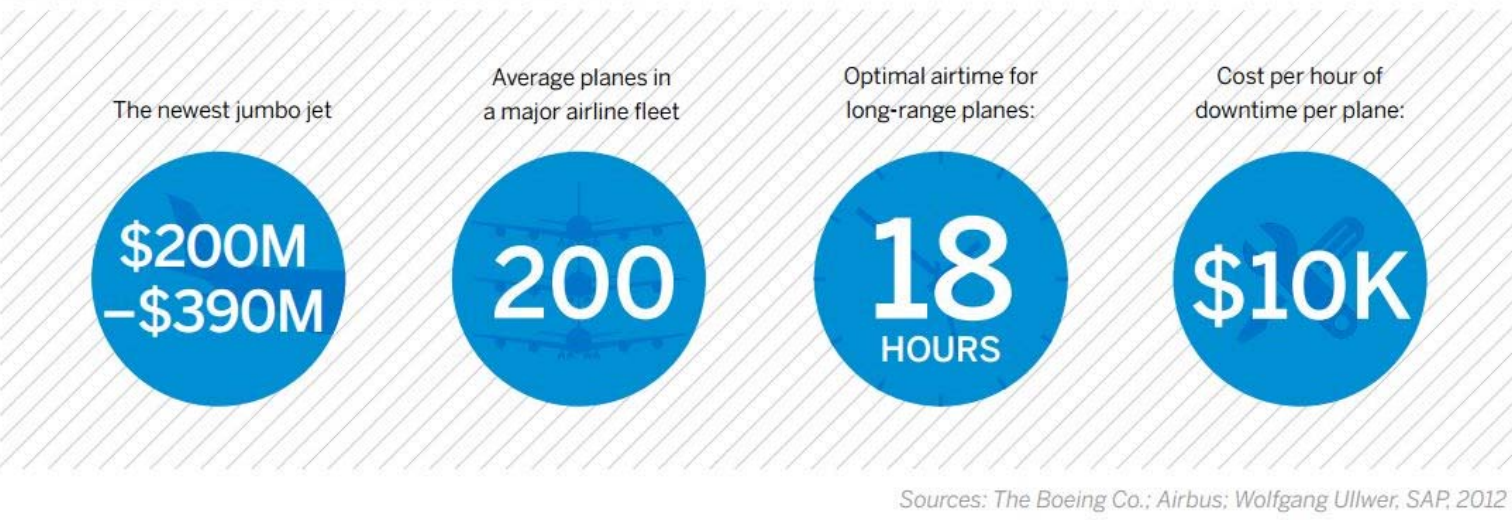

Fig. 3. Why Airlines need to keep planes in the air [2]

Predictive analysis can help convert huge amounts of relevant maintenance data (whether from sensors or logistics information from ERP systems - Enterprise Resource 
Planning) into information that can be taken to ensure that maintenance technicians perform the steps match at the right time and with the right tools. The anticipative analysis can contribute to strategic improvement and also to delivering better quality production with lower operating costs and increase the investment.

\section{The need to analyze high volume data}

Beneficiaries of high-volume data analysis are represented by: air traffic controllers, airlines, aircraft manufacturers, etc. With data provided by aircraft, on flight or on the ground, the beneficiaries can optimize their operational efficiency, improving the company's operations, aircraft production, timely maintenance planning, etc. Examples of efficient use / analysis of high volume data:

- congestion of air traffic can be reduced;

- passengers may be informed about timetables and flight operations on their way to the airport;

- air traffic delays can be reduced;

- scheduled maintenance work, avoiding additional costs.

These are just a few things that would simplify the flights or company activities. However, airlines and airports still have limited capabilities to process / implement this valuable information quality and to use advanced systems to analyze large volume data in order to optimize information.

All of these beneficiaries must provide aircraft for the pilots in perfect working order to meet in good flight conditions. Although the analysis of large volume data is complex and working conditions are not always the most favorable, the satisfaction of maintaining the maximum operational capacity of aircraft is very high.

There are, however, a lot of airline maintenance organizations where large data analysis, Big Data, is not being implemented, all based on a production plan on the basis of which, at the beginning of the year, there is just a need for spare parts and material, but which is not entirely secured. Unpredictable malfunctions occur during the year, they are more difficult to repair due to shortages and, following reports of necessity, technicians have to take the from other aircraft, which leads to the delay in finalizing the works, doubling the effort of the technical staff, thus involving additional costs.

Currently, an aircraft that performs a flight, has its own flight plan (a series of points over which it will pass to reach the destination). In the vast majority of cases, it does not happen as the flight is planned because there are a number of events that lead to this conclusion, such as:

- the airline has shifted the departure time for an aircraft, for various reasons;

- a meteorological event forbids any aircraft to pass through a certain area;

- a traffic controller gives the pilot permission to use a "shortcut".

The use of high volume data analysis for the benefit of air traffic control can be used to manage air traffic safely, create more efficient flight routes, and exchange information with airlines to improve performance and flight safety.

\section{Case studies using Big Data}

\subsection{Real-time aircraft monitoring using the}

Big Data platform - SAP Predictive Maintenance

Maintenance is the repair, inspection or modification of an aircraft or its component. Aircraft maintenance is regulated to ensure safe and correct flight operation of the aircraft. National regulations are coordinated in line with international standards, maintained by bodies such as the International Civil Aviation Organization (ICAO). 


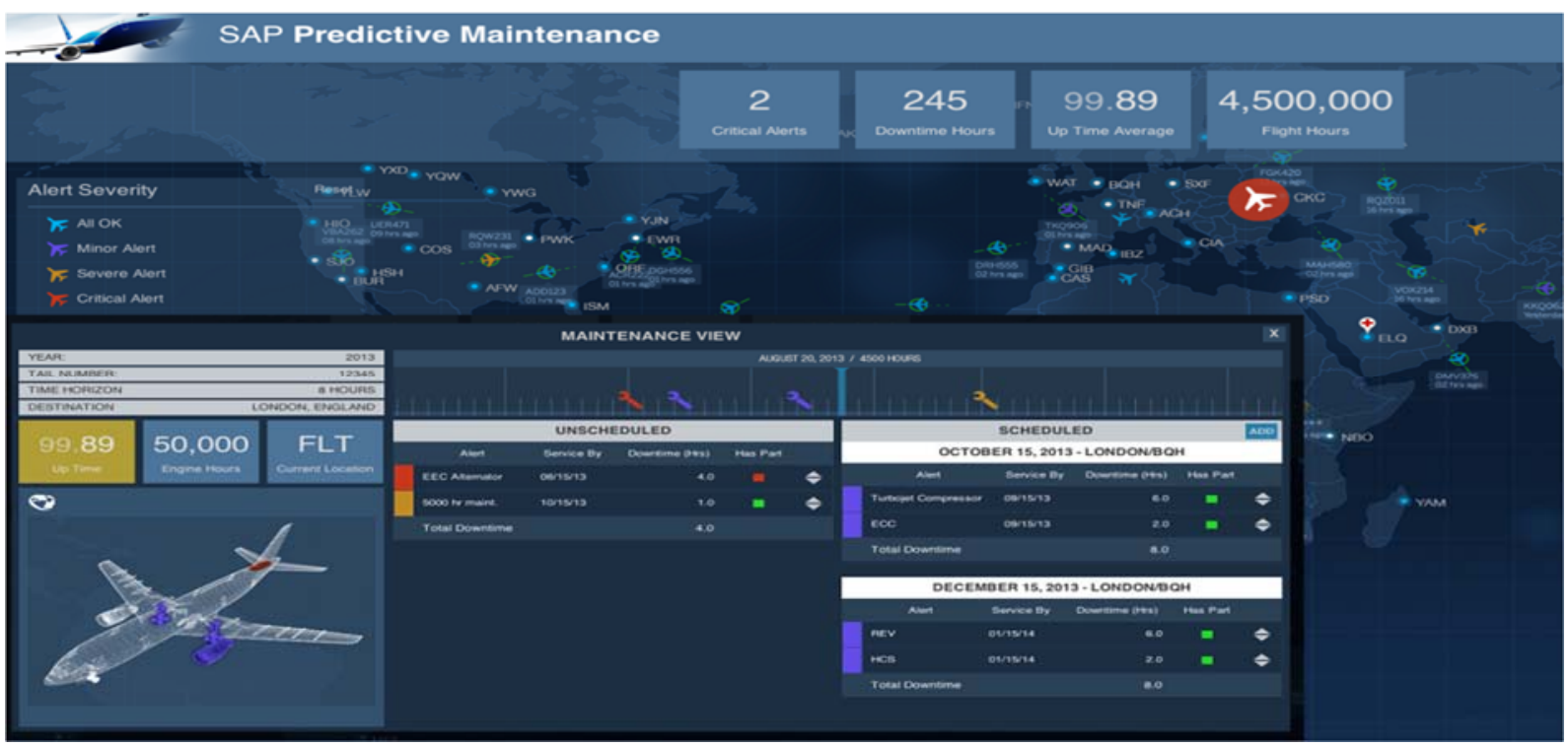

Fig. 4. SAP Predictive Maintenance [3]

Predictive Maintenance Technique is designed to help determine the loss of an aircraft equipment and to predict when maintenance is to be done.

The key to predictive maintenance is "correct information at the right time"

In the above Figure 4, with the help of the SAP Predictive Maintenance program, the Big Data from an aircraft in flight is analyzed in real time.

Figure 4 gives us the following information: the aircraft have to make in October the scheduled maintenance for the engine compressor, also we can see that the repair time is 6 hours, and the replacement part is in stock. As unscheduled maintenance, the airplane has to replace an engine electrical component - EEC, the repair time is 4 hours, but the piece is not yet in stock. As well as unscheduled maintenance, the aircraft has to perform the necessary maintenance after 5,000 hours of flight, this operation can be seen it will take an hour.

\subsection{Real-time monitoring of aircraft propulsion systems (engines)}

Air transport is necessary for modern life. Maintenance of aircraft propulsion systems is costly, requiring a very careful approach.

Propulsion systems are equipped with sensors that monitor the engine's operating conditions during the flight. In the figure below are a few sensors that fit an aircraft engine.

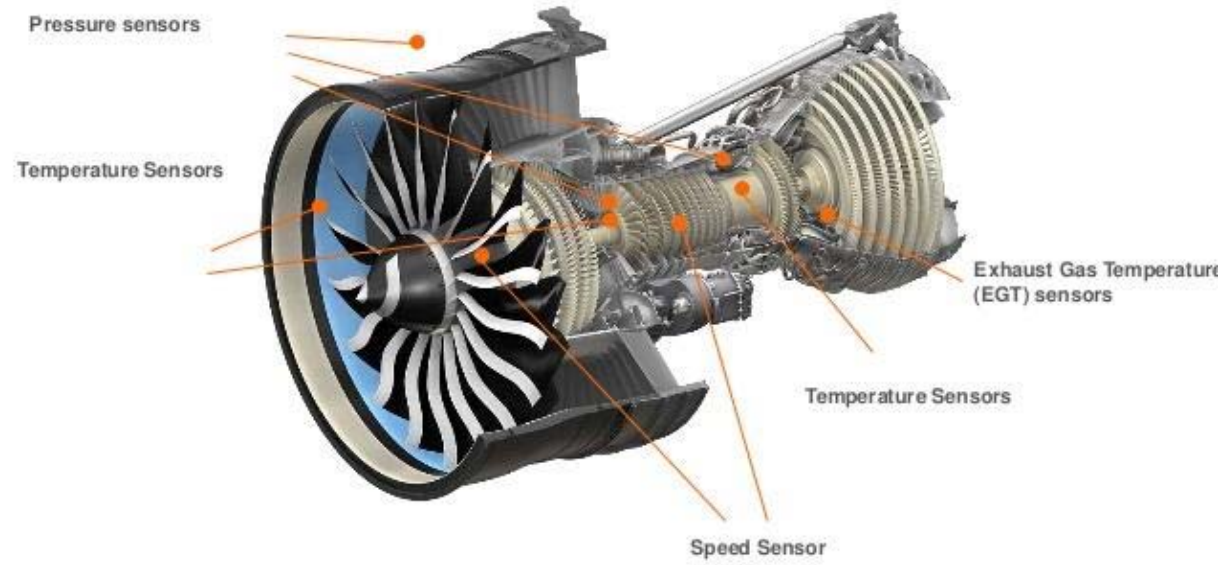

Fig. 5. Simplified view of some of the sensors [4] 
By combining data from these Big Data Sensors with advanced analyzes, it is possible to monitor the behavior of propulsion systems in real time, so that we can predict the remaining useful life of a component belonging to the engine so we can schedule maintenance in a timely manner to avoid unnecessary mechanical malfunctions, but also a very high cost generated by defective maintenance.

This monitoring system includes data entry, data storage, data processing and analysis, all of this are essential for creating a predictive maintenance solution.

Although this example is customized for aircraft engine monitoring, the solution can be easily generalized for other predictive maintenance scenarios such as landing gear, structural integrity of the aircraft, etc.

By reducing downtime and ensuring effective engine performance, this solution allows the aircraft fleet to remain operational in the most cost effective way.

If an aircraft is in flight, poor maintenance of propulsion systems can have very serious consequences. The best case may be to land the aircraft after a failure in the propulsion system, in a safe condition, until the malfunction is remedied. This involves problems that can be particularly costly, given that the aircraft is in a location where there are no suitable technicians or spare parts.

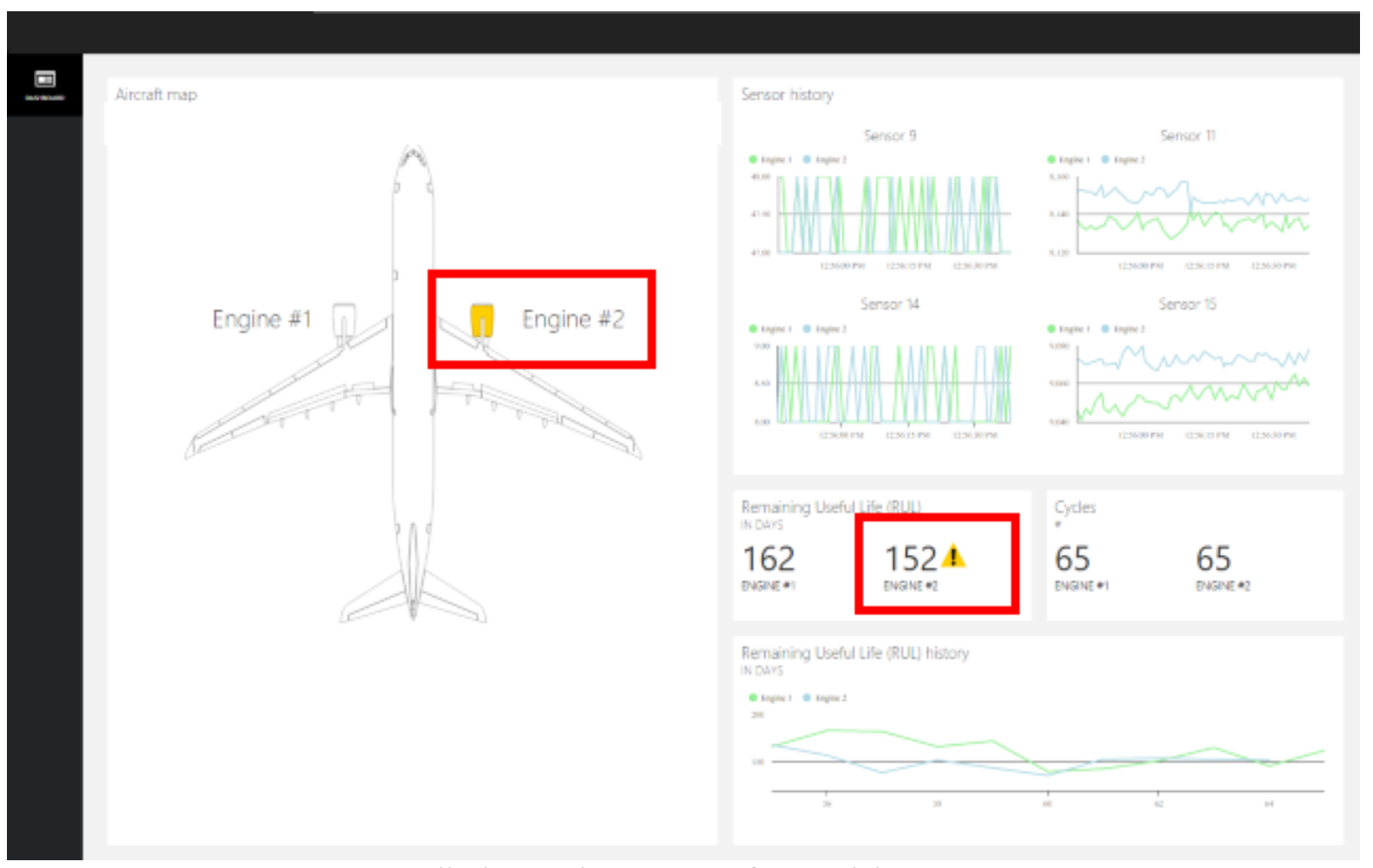

Fig. 6. Predictive maintenance of propulsion systems [5]

Figure 6 is based on the following input data: both engines have a number of 4 sensors, namely: temperature sensor, pressure sensor, noise level sensor, vibration level sensor. The analysis is performed to determine the remaining useful life of the engines that fit this aircraft. This analysis is based on a number of 65 cycles per engine. The higher the number of cycles, the greater the efficiency of the data provided. Following this predictive maintenance analysis of propulsion systems based on high volume data analysis, we draw the following conclusions: By analyzing the data from the four sensors, we can easily see that the engine with no. 2 which equips this aircraft, has a less useful life than the engine with no. 1, 152 days versus 162 days. This 
analysis is very useful for the maintenance organization's technicians, because they know exactly when both engines must be out of service, so it can be timely scheduled the maintenance required to replace it, or replacement of both engines, given that the remaining useful life for the two engines is near, so that the aircraft is not immobilized twice for the same operation, resulting high efficiency and low cost.

\section{Conclusions}

High volume data start to have a major impact on air travel. The opportunities to use these data will continue to grow in the future. An airplane is more than just a classic car that takes us from one city to another. Modern airplanes are data centers themselves. The analysis of high volume data for an airline starts from aircraft predictive maintenance, flight plans, weather conditions and up to the purchase of tickets by future passengers. For an airline or even an air traffic controller, the weather conditions are a key element in ensuring a safe flight. So, combining two different data sources (weather forecasts and flight plans), the users of the high volume data analysis can view in real time the places where the flight can intersect with some unfavorable meteorological conditions, giving more time to carry out of alternative plans.

Each element of a plane's performance is monitored in real-time to make adjustments that significantly reduce maintenance costs and, more importantly, save lives by improving flight safety.

The main advantages of using large volume data analysis:

- low maintenance costs and improved aircraft availability through optimization of maintenance program;

- low inventory requirements for parts requiring replacement through the integrated distribution chain and planning;

- fuel economy;

- optimizing flight plans.

The commercial impact of implementing high-volume data analysis has the potential to generate a significant increase in productivity and optimization in many key sectors. High volume data analysis provides the opportunity to create unprecedented business benefits and to provide better services to beneficiaries. Managing and analyzing high volume data in real time data is not just a storage or visualization process, it's the ability to make better decisions and take meaningful measures at the right time.

\section{Acknowledgement}

This work was supported by the Institutional research programme PN 1819 "Advanced IT resources to support digital transformation processes in the economy and society RESINFO-TD" (2018), project PN 1819-0101 "New research in complex systems modelling and optimization with applications in industry, business and cloud computing", funded by the Ministry of Research and Innovation.

\section{References}

[1] A. Alexandru, D. Coardos, Big Data, Concepte, arhitecturi si tehnologii, Revista Romana de Informatica si Automatica, vol.27, nr. 1, 2017

[2] https://blogs.sap.com/2015/01/20/whyairlines-need-to-keep-planes-in-the-air/

[3]

https://blogs.sap.com/2014/12/08/comme rcializing-big-data-opportunities-inaerospace-and-defense/

[4] https://rolls-

[5] https://docs.microsoft.com/enus/azure/iot-suite/iot-suite-predictiveoverview

[6] R. Boncea, I. Petre , D.-M. Smada, A. Zamfiroiu, A Maturity Analysis of Big Data Technologies, Informatica Economică vol. 21, no. 1/2017.

[7] K. E, Prasanna Devi, V.S. Kiran, S. Mannivannan, Airline Route profitability analysis and Optimization using BIG DATA analyticson aviation data sets under heuristic techniques, Procedia Computer Science, volume 87, 2016

[8] M. Pazovrek, V. Vaclavik, Assessment of Business Aviation OCC's Capacity Issues, Procedia Engineering, volume 
187,2017

[9] Y. R. Zhong, T. Stephen. G. Newman, Q. Huang, S. Lan, Big Data for supply chain management in the service and manufacturing sectors. Challenges, Opportunities and future perspectives, Computer \& Industrial Engineering, volume 101, 2016

[10] A. Gunasekaran, T. Papadopoulos, R. Dubey, S.F. Wamba, S. J. Childe, B.
Hazen, S. Akter, Big Data and predictive analytics for supply chain and organizational performance, Journal of Business Research, volume 70, 2017

[11] International Air Transport Association (IATA), Annual Review, 2017

[12] International Civil Aviation Organization (ICAO), Making Borders More Secure, The Benefits of Automation, ICAO TRIP, volume 12, 2017

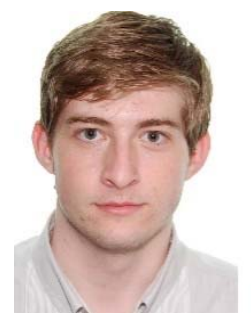

Emmanuell BADEA has graduated the Faculty of Aerospace Engineering Propulsion Systems in 2015. In 2017 he has graduated the Aerospace Propulsion and Environmental Protection - Master Program at the same Faculty of Aerospace Engineering. In 2018 he joined the staff of the National Institute for Research and Development in Informatics, as aerospace engineer. He's interested in use of computer codes and of the digital technologies for solving tasks specific to aerospace engineering, particularly in gas dynamics and calculation of propulsion systems components.

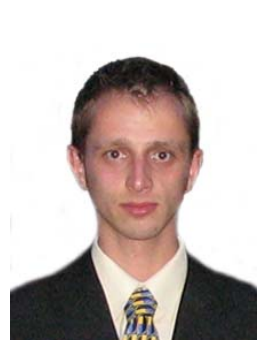

Alin ZAMFIROIU has graduated the Faculty of Cybernetics, Statistics and Economic Informatics in 2009. In 2011 he has graduated the Economic Informatics Master program organized by the Academy of Economic Studies of Bucharest and in 2014 he finished his PhD research in Economic Informatics at the Academy of Economic Studies. Currently he works like a Senior Researcher at "National Institute for Research \& Development in Informatics, Bucharest". He has published as author and co-author of journal articles and scientific presentations at conferences.

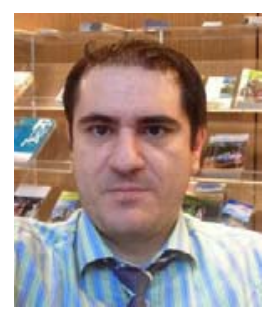

Radu BONCEA is a Researcher at I.C.I. Bucharest. He has been involved in several large European projects such SPOCS, eSENS, Cloud for Europe and The Once Only Principle. As a Ph.D. student at Electronics, Telecommunications and Information Technology, he's interested in IoT and Cloud Computing related technologies. 